\title{
Adaptación de los dominios científicos, tecnológicos y humanísticos en la academia a causa del COVID-19
}

Fecha de recepción : 2020-04-20 • Fecha de aceptación: 2020-09-10 • Fecha de publicación: 2021-10-01

Florcita Janeth Arellano Espinoza ${ }^{1}$

Universidad Estatal de Bolívar, Ecuador

farellano@ueb.edu.ec

https://orcid.org/0000-0002-4325-9317

María de los Ángeles Bonilla² Universidad Estatal de Bolívar, Ecuador mabonilla@ueb.edu.ec https://orcid.org/0000-0003-2051-4626

Jonathan Patricio Cárdenas Benavides ${ }^{3}$ Universidad Estatal de Bolívar, Ecuador jcardenas@ueb.edu.ec https://orcid.org/0000-0002-9760-602X

Danny Fernando Pérez Castillo ${ }^{4}$ Universidad Estatal de Bolívar, Ecuador dperez@ueb.edu.ec https://orcid.org/0000-0003-4418-0050

\section{Resumen}

Los dominios universitarios son sistemas complejos de conocimientos tecnológicos, humanísticos y científicos que se integran en las instituciones de educación superior a sus saberes, cosmovisiones, a sus prácticas culturales y sociales. El objetivo del estudio es conocer los dominios científicos, tecnológicos y humanísticos de las instituciones de educación superior del Ecuador. La investigación cuenta con una metodología de enfoque cualitativo de tipo descriptivo. Una de las conclusiones es 
que estos aportan como ejes a la planificación estratégica institucional, y ayudan a los procesos sustantivos como es la docencia, investigación y vinculación de las instituciones hacia los diversos problemas que se pueden presentar. La pandemia de COVID-19 ha obligado a la mayoría de las instituciones educativas a la modalidad obligatoria de clases en línea. Para realizar el ajuste, las instituciones han optado por el uso de herramientas tecnológicas, donde el principal involucrado es el educador, por este motivo, los dominios se han involucrado de forma distinta.

Palabras clave: competencias docentes, COVID-19, dominios universitarios, pedagogía emergente, educación superior.

\begin{abstract}
The university domains are complex systems of technological, humanistic, and scientific knowledge that are integrated into higher education institutions, their knowledge, worldviews, and cultural and social practices. The objective of the study is to know the scientific, technological and humanistic domains of Ecuador's higher education institutions. The research has a descriptive qualitative approach methodology. One of the conclusions is that these contribute as axes to institutional strategic planning being of help to the substantive processes such as teaching, research and linking of institutions towards the various problems that may arise. The COVID-19 pandemic has forced the majority of educational institutions to the compulsory modality of online classes. To make the adjustment, the institutions have opted for the use of technological tools, where the main involved are educators, for this reason the domains have been involved in a different way.
\end{abstract}

Keywords: teaching competencies, COVID-19, university domains, emerging pedagogy, higher education 


\section{Introducción}

Las instituciones de Educación Superior (IES) basan su accionar en tres ejes principales que son las funciones sustantivas de: docencia, investigación y vinculación con la sociedad, agregando a ellas el eje de condiciones institucionales. Estas funciones están organizadas en relación con su planificación, ejecución y resultados alcanzados a través del tiempo (LOR LOES, 2018); las instituciones de educación superior prestan servicios de educación de manera privada y pública.

La colectividad se vincula inmersa a las funciones fundamentales en la educación superior, ya que cada universidad se encuentra relacionada a su base administrativa, junto a su misión, visión, realidad del sector, políticas y reglamentos que la imperan (Bonilla et al., 2019); ésta función sustantiva es la que refleja la eficiencia y eficacia de los dominios científicos, tecnológicos y humanísticos que cada IES posee.

El objetivo del presente estudio es conocer los dominios científicos, tecnológicos y humanísticos de las instituciones de Educación Superior del Ecuador que fundamentan la planificación estratégica de las universidades, relacionándose de forma directa con la profesión, investigación, innovación y vinculación. Es importante destacar los cuerpos polisémicos y la importancia de trabajar en equipos interdisciplinarios y transdisciplinarios que funcionan en ecosistemas organizacionales; aquí, es donde se debe hablar del principio de pertinencia que está reflejado básicamente en la LOES (2010), artículo 107 y expresa, que las universidades respondan a las expectativas y necesidades de la sociedad, al plan nacional de desarrollo, a la prospectiva de progreso científico, humanístico, tecnológico y mundial.

Estos dominios se convierten en un eje directo de la planificación estratégica y de las funciones de investigación, formación y vinculación. Las mencionadas instituciones cumplen una misión estratégica en nuestra generación con respecto a la innovación tecnológica y social, misma que se consolida en la articulación de las diferentes plataformas interinstitucionales. Como componentes se encuentran: las redes de innovación y prospectiva, la capacidad y potenciales de las instituciones, las áreas de priorización, las matrices estratégicas, los conocimientos, y por supuesto, las tecnologías y saberes.

En el Ecuador, con la actual pandemia, se modernizan las líneas de acción alrededor de las universidades, promoviendo la movilidad en instituciones de investigación y formación, la cooperación por la calidad desarrollando metodologías y comparación de criterios, junto a las funciones sustantivas establecidas en la LOES a través de la virtualidad. El reintegrarse a las clases universitarias en Ecuador se hará en forma progresiva, por etapas, hasta llegar a su forma presencial o semipresencial y con plataformas online, con estricto control de bioseguridad y con el aforo estudiantil necesario para la prevención del COVID-19 (enfermedad infecciosa causada por el coronavirus).

Se ha examinado el desarrollo y ecosistema digital en América Latina y el Caribe en relación con diversas regiones mundiales, lo que ha provocado un estancamiento en el desarrollo educativo, con el incremento de espacios de equidad y calidad detallados con indicadores porcentuales. Esto significa que el Consejo de Educación Superior (CES) ha determinado normativas que repercuten 
en nuevos dominios científicos, tecnológicos y humanísticos donde se hablan de los manejos de dominios tecnológicos, adoptando la modalidad híbrida que garantiza la continuidad del estudio del alumnado. Las IES ofrecerán a los estudiantes acceso abierto a por lo menos una biblioteca virtual y un repositorio digital y guías de estudio para cada asignatura, curso o su equivalente por si el estudiante no tiene acceso a medios tecnológicos, este podrá acceder al material del curso y desarrollar sus actividades académicas mediante el aprendizaje autónomo.

El modelo de evaluación externa agrupa una doble definición de innovación. Propende a que las universidades y escuelas politécnicas contribuyan con actividades de investigación y vinculación con la sociedad. Se menciona el régimen académico y su reglamento, que responde a expectativas académicas y se potencializa el principio de pertinencia. Las funciones universitarias se encuentran articuladas a sectores y ejes estratégicos, tal como se mencionan en el objetivo No. 5 de la Secretaría Nacional de Planificación y Desarrollo (2017). Las fortalezas y dominios universitarios que evolucionaron con excelencia en las instituciones de nivel superior que originaron en el nacimiento del CES con una acreditación especial de la planificación estratégica para lograr sus indicadores.

En este contexto, sin duda, se hace necesario definir ciertos términos. Un dominio es una red compleja, polisémica, cambiante e histórica de gestión y generación pertinente e importante del conocimiento en aspectos culturales, tecnológicos y científicos, la estructura se encuentra centrada en redes científicas y académicas generadas por las necesidades de territorios, zonales, locales, nacionales, regionales e incluso mundiales (Ruzmar \& Pérez, 2017).

Los dominios se han convertido en una propuesta de organización y formación de contenido y conocimiento debido a la gestión dialógica de la diversidad de aspectos culturales, sociales y productivos, los cuales actúan en la responsabilidad social, beneficiando de forma directa al desarrollo de estrategias y de la autentificación de las instituciones de educación superior, generando así un conocimiento distribuido, abierto, solidario, intercultural, participativo y justo (Larrea \& Granados, 2013).

A su vez, los organismos polisémicos son cuerpos interdisciplinarios y transdisciplinarios. Se manejan bajo una dinámica organizativa, la cual se encuentra direccionada al fortalecimiento de las capacidades contextualizadas, diferenciadas y de un nivel elevado de polivalencia de las universidades; permiten la integración hacia redes del sistema y hacia la articulación con los entornos académicos, productivos, sociales, ambientales y culturales.

En lo definido por Ramírez (2016), señala que estos dominios generan nuevas disposiciones epistemológicas, disposiciones que se reflejan en visiones orgánicas inclusivas y democráticas, considerando la integración de la misión sustantiva de las universidades y refiriéndose a derechos, deberes y prospectivas del proyecto de sociedad planteado en el programa del Buen Vivir, mediante las prácticas científicas y académicas, para que estas aporten a la generación del conocimiento y a los aprendizajes sociales y tecnológicos.

En el título VI de la Ley de Educación Superior se establece en el capítulo 1 del principio de pertinencia en donde el artículo 107 describe lo siguiente: 
"El principio de pertinencia consiste en que la educación superior responda a las expectativas y necesidades de la sociedad, a la planificación nacional, y al régimen de desarrollo, a la prospectiva de desarrollo científico, humanístico y tecnológico mundial, y a la diversidad cultural. Para ello, las instituciones de educación superior articularán su oferta docente, de investigación y actividades de vinculación con la sociedad, a la demanda académica, a las necesidades de desarrollo local, regional y nacional, a la innovación y diversificación de profesiones y grados académicos, a las tendencias del mercado ocupacional local, regional y nacional, a las tendencias demográficas locales, provinciales y regionales; a la vinculación con la estructura productiva actual y potencial de la provincia y la región, y a las políticas nacionales de ciencia y tecnología" (CEAACES, 2018 p. 30).

Los dominios de las IES del Ecuador serán el eje directo de la planificación estratégica y de las funciones de investigación, formación y vinculación. Se apertura así la oportunidad de generar las articulaciones con sectores y actores de desarrollo (Maldonado, 2017). Por lo descrito anteriormente, se comprende que los dominios y su planificación aseguran la pertinencia y la calidad de la gestión universitaria, incrementando los beneficios si el eje se encuentra direccionado a la producción de la investigación con base en la epistemología. Del mismo modo, se vuelve imprescindible, formar talento humano y acrecentar la responsabilidad social, denominada como vinculación universitaria. Se menciona la inadaptabilidad de los maestros en las plataformas virtuales, pese a la previsión de los recursos tecnológicos y donde la vinculación con la sociedad no es posible por no tener acceso a la movilidad en estudiantes y docentes hacia los sectores de la entidad. Los dominios científicos, tecnológicos y humanísticos examinan la gestión de autoridades y docentes para transformar sus debilidades en fortalezas y potencializar eficientemente sus áreas de acción; es momento entonces, de la transformación de la educación superior y de que se aprovechen los recursos tecnológicos para que se acoplen a las estructuras funcionales en el proceso de gestión de la información.

Las IES mantienen su misión estratégica en la generación e innovación tecnológica y social, la misma que se consolida en el momento de la articulación con las diversas plataformas interinstitucionales, así como también, en las orientaciones hacia las ciencias, humanidades y tecnología con las necesidades que surgen del conocimiento, lo que provoca la integración de los ciudadanos en la distribución de proyectos en áreas como investigación, innovación o desarrollo en el momento en el que las universidades enlazan las funciones sustantivas con la finalidad de trabajar en servicio de la resolución de los problemas que se presentan en la sociedad (Pineda, 2013). En base a lo descrito anteriormente se estructura la planificación por dominios, ver Figura 1. 


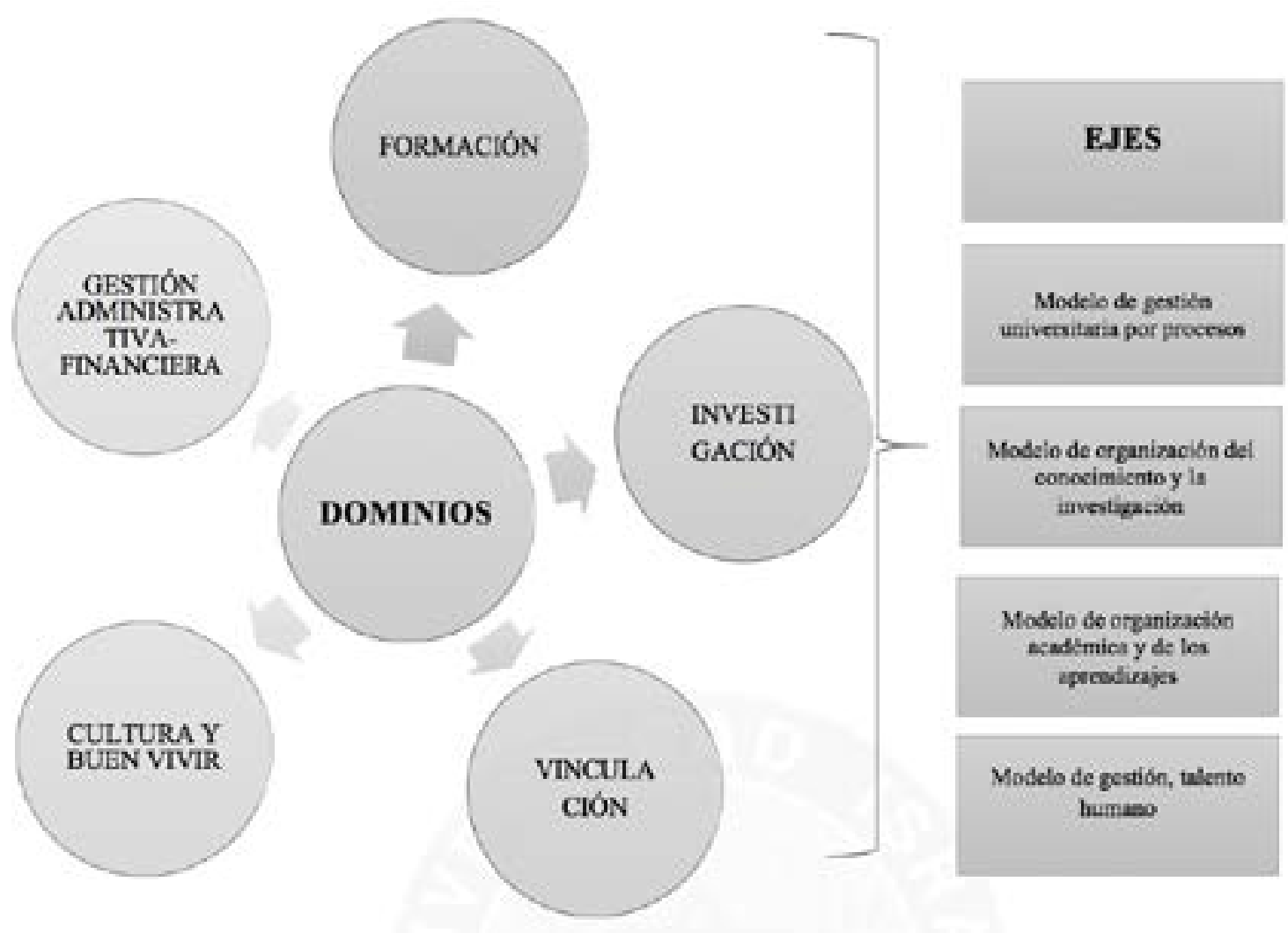

Figura 1. Planificación por dominios universitarios

Fuente: Información obtenida de "Modelo de organización del conocimiento por dominios científicos, tecnológicos y humanísticos” (Larrea \& Granados, 2013).

Los dominios universitarios cuentan con varios componentes entre los que se encuentran: a) redes de innovación y prospectiva; b) capacidad y potenciales de las instituciones; c) áreas de priorización y matrices estrategias y d) conocimientos, tecnologías y saberes.

En Ecuador se han modernizado las líneas de acción en el marco de las universidades, promoviendo la movilidad en instituciones de investigación y formación mediante la cooperación para la calidad con el desarrollo de metodologías y criterios comparables, que comulgan con las funciones sustantivas establecidas en la LOES, dirigidas a establecer una academia más completa y de mayor alcance, construida en particular, mediante el desarrollo y fortalecimiento de los dominios científicos, tecnológicos y humanísticos (Larrea \& Granados, 2013). El estado de excepción decretado por la emergencia sanitaria ocasionada por la pandemia de COVID-19, develó las deficiencias existentes que venían arrastrando desde años anteriores las IES. Se evidenció de forma más clara (ver Figura 2), la inadaptabilidad en el uso de plataformas tecnológicas, y la limitada solución en problemas de contexto cultural, social, productivo. 


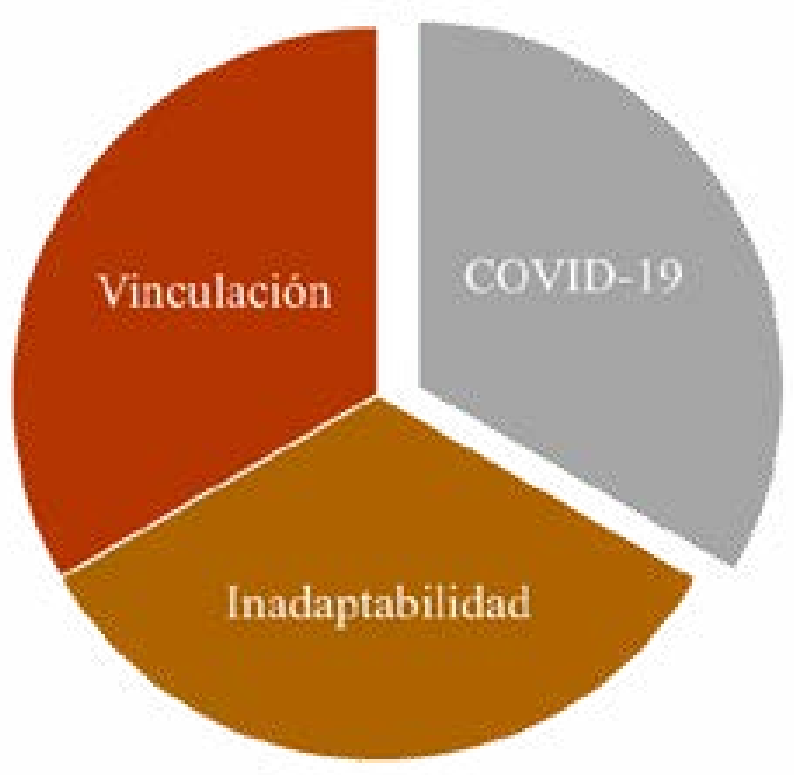

Figura 2. Problemas actuales de los dominios universitarios

Fuente: elaboración propia

El nivel 1 que aparece fuera de la Figura 2 enfatiza los efectos que ocasiona el COVID-19 con respecto a dos problemas relevantes. El primero es de inadaptabilidad por parte de los docentes a las plataformas virtuales a pesar que existe una alta previsión de los recursos tecnológicos. Como segundo punto se encuentra la vinculación con la sociedad que por el impedimento de la movilidad de docentes y estudiantes hacia la transformación de los sectores en los que se desarrolla la academia. Es en este mismo contexto, donde los dominios científicos, tecnológicos y humanísticos deben analizar la gestión de autoridades y docentes para convertir estas debilidades en fortalezas y potencializar las áreas de acción. Por el momento se evidencia la falta de prevención de sistemas informáticos, organización tecnológica y presupuestos necesarios para afrontar decididamente esta crisis sanitaria. Algunas universidades públicas se encuentran desarmadas en plataformas tecnológicas, a diferencia de un buen número de universidades privadas que están superando esta fase, gracias a su previsibilidad y estructuración de la planificación estratégica y prospectiva de los procesos sustantivos, que conducen a la gestión estratégica de excelencia académica y permite afrontar esta crisis.

En este tiempo de transformación de la educación superior se deben aprovechar los recursos tecnológicos para adaptar estructuras funcionales al proceso de gestión de la información. Se trata de experimentar acciones que ayuden a la optimización del tiempo y al incremento de resultados, partiendo de la palabra clave "adaptación", que significa, romper la resistencia al conocimiento y capacitación del adecuado uso de las pestañas, herramientas, navegadores, sistemas de calificaciones y asignación de tareas, para entender que los resultados mejoran con el uso de las tecnologías de información y comunicación, encontrándose al alcance de todos, abriéndose 
a fomentar los entornos digitales educativos formales, obteniendo ventaja en tiempo y espacio académico (Mora et al., 2018).

La reinserción de las clases universitarias en Ecuador se efectuará de manera progresiva y por etapas, hasta regresar a ser presencial y en algunos casos semipresencial, respetando el aforo en las aulas y cumpliendo normas de bioseguridad para evitar posibles contagios del COVID-19; en cambio que, para las nuevas carreras online, se busca implementar plataformas virtuales, realizando capacitaciones completas dirigidas a autoridades, docentes y estudiantes, observándose en todo el proceso, las perfecciones y deficiencias existentes. La pandemia obligó a la educación superior a que la transición hacia un nuevo modelo educativo sea abrupta, lo que representa un duro desafío para los integrantes del sistema académico y un desafío mayor a superar, debido a la desigualdad social en que vive América Latina (Rojo \& Bonilla, 2020).

América Latina y el Caribe están posicionados en un nivel de desarrollo intermedio con respecto a otras regiones del mundo en términos de desarrollo de su ecosistema digital. Esto provoca un estancamiento sobre el desarrollo educativo, aumentando las brechas de equidad y calidad; esta región tiene un índice de 49.92 (en una escala de 0 a 100), permaneciendo en una posición más avanzada en comparación con África (35.05) y Asia del Pacífico (49.16). Sin embargo, pesar de los avances significativos de los últimos 15 años en el desarrollo de su ecosistema digital, América Latina y el Caribe todavía muestra un rezago respecto a Europa Occidental (con un índice de 71.06), América del Norte (80.85), Europa del Este (52.90) y los Estados Árabes agrupados en torno al acrónimo Mena (55.54) (CAF, 2020).

EI CES reacciona inmediatamente y determina normativas que repercuten en los nuevos dominios científicos, tecnológicos y humanísticos en las universidades, especialmente en el artículo 4a y $4 \mathrm{~b}$, que habla sobre las nuevas disposiciones del manejo de dominios tecnológicos para las universidades, al expresarse singularmente sobre el cambio de modalidad de clases. Adopta la modalidad híbrida para garantizar la continuidad de estudios del alumnado, las IES podrán adaptar sus planes de carreras y programas a la mencionada modalidad, que es un método alternativo de enseñanza que surge con estas nuevas tecnologías educativas y muestra eficiencia en varios contextos de aprendizaje, especialmente solucionando problemas de los estudiantes que tienen dificultad de recibir la clase expositiva tradicional de manera presencial.

Esta modalidad de enseñanza priorizará el aprendizaje autónomo de los estudiantes, para lo que se requiere que todo curso, asignatura o su equivalente contenga una guía de estudios desarrollada por el personal académico. Para fortalecer los procesos de aprendizaje autónomo de los estudiantes, así como la ampliación, profundización y especialización de los conocimientos, las IES deberán ofrecer al estudiante el acceso abierto al menos a una biblioteca virtual y un repositorio digital de apoyo y las guías de estudio deben garantizar la continuidad de los estudios del alumnado.

Las IES elaborarán guías de estudio por cada asignatura, curso o su equivalente, de tal forma que el estudiante que no tenga acceso a medios tecnológicos pueda acceder al material del curso y desarrollar las actividades académicas de la asignatura mediante el aprendizaje autónomo. Las guías contendrán como mínimo los objetivos de la asignatura, curso o su equivalente, contenidos, 
actividades de aprendizaje, parámetros y actividades de auto y heteroevaluación (preferiblemente por temas o unidades didácticas), bibliografía básica y complementaria. Dichas guías estarán en formato digital descargable para su fácil acceso, y en formato impreso, en el caso de que los estudiantes justificadamente así lo soliciten. Las guías deberán ser entregadas al estudiante al inicio de cada período académico y reemplazarán al sílabo (Normativa transitoria para el desarrollo de actividades académicas en las Instituciones de Educación Superior. RPC-SE-03-No 046-2020, 2015). A continuación, se muestra la Figura 3 con los bloques de información agrupada.
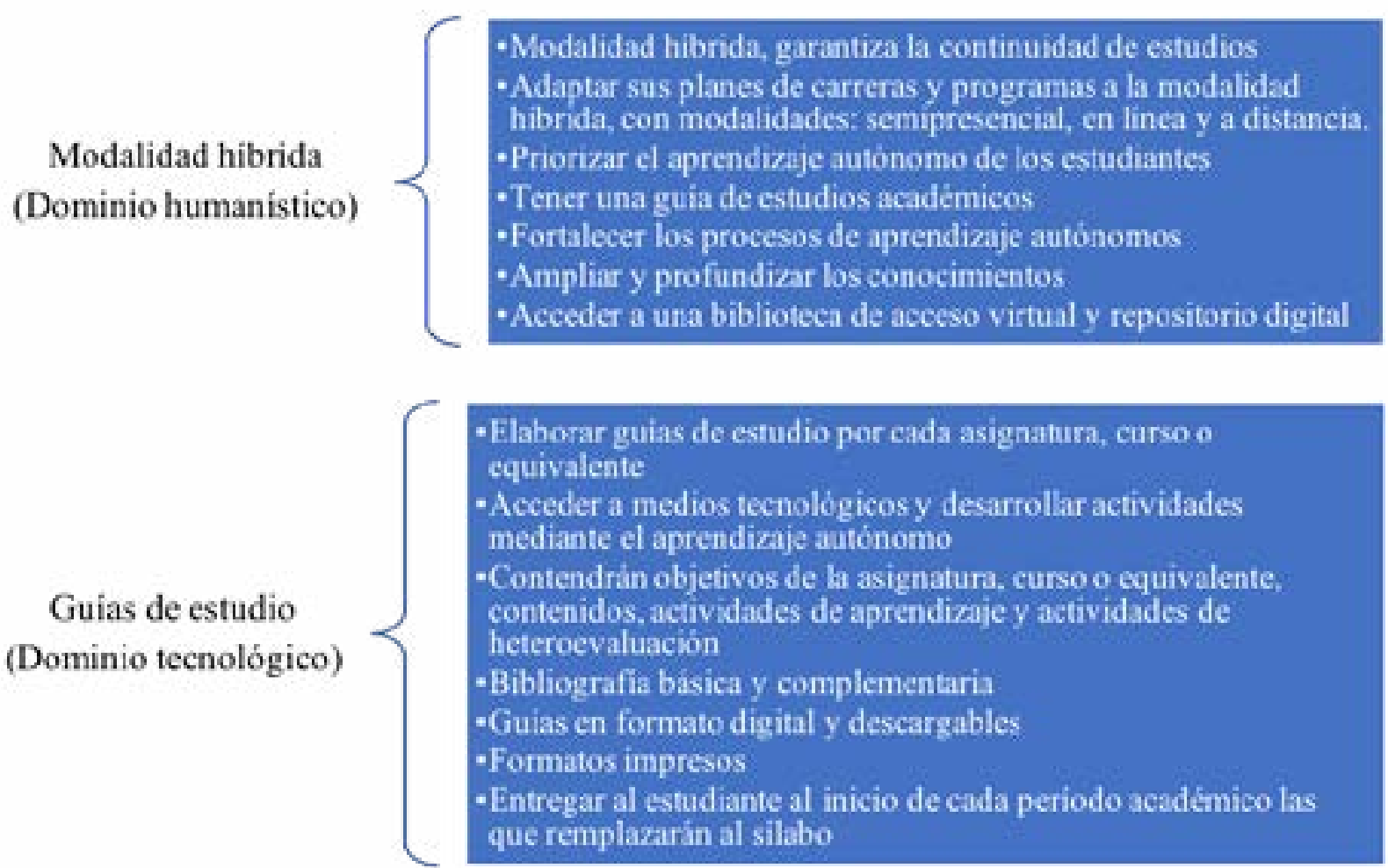

Figura 3. Artículos 4a y 4b, cambios de modalidad en las actividades académicas planificadas. Fuente: Normativa transitoria para el desarrollo de actividades académicas en las Instituciones de Educación Superior. RPC-SE-03-No 046-2020

El último modelo de evaluación externa propone una doble definición de innovación en relación con la contribución que hacen las universidades y escuelas politécnicas a la sociedad a través de la innovación que desarrollan como parte de sus actividades de investigación y de vinculación con la sociedad, y también en relación con las prácticas innovadoras que desarrollan las IES en su interior y que son transversales a todas las funciones sustantivas y a su gestión.

El régimen académico y su reglamento tiene la responsabilidad de responder a las expectativas y necesidades del entorno académico dependiendo de la ciudad y región; potencializando el principio de pertinencia, ciñéndose“... a la prospectiva de desarrollo científico, humanístico y tecnológico mundial, y a la diversidad cultural" (LOES, 2010). Los dominios universitarios son 
comprendidos como sistemas complejos de conocimientos tecnológicos y científicos, que se integran a las instituciones de educación superior y a sus saberes, cosmovisiones y a sus prácticas culturales y sociales (Rivadeneira, 2016), por ello se viabiliza las unidades de las funciones universitarias que se encuentran articuladas con los sectores y ejes estratégicos, conforme al objetivo No. 5 del Plan Nacional de Desarrollo 2017-2021 que impulsa la productividad y competitividad para el crecimiento económico sostenible de manera redistributiva y solidaria, en donde se formulan metas que integran a las IES al intercambio de estos dominios para poder alcanzarlos; estas metas, por ejemplo, se basan en incrementar el porcentaje de la inversión en investigación y desarrollo como porcentaje del Producto Interno Bruto del 0,44\% al 0,48\% a 2021 o incrementar de 78 a 153 el número de solicitudes de patentes nacionales al 2021, y especialmente el aumento del número de publicaciones científicas al 2021 (SENPLADES p. 83, 2017).

El tema de las fortalezas y dominios universitarios pronostica una mejora obligatoria de la evolución de la excelencia de las instituciones de educación superior que se ha trabajado desde el nacimiento del CES y su alcance en los niveles y modelos de evaluación calidad y acreditación expresados a través de la planificación estratégica en las instituciones para el cumplimiento y logro de los indicadores (Morales, 2012).

\section{Metodología}

El presente artículo toma una ruta cualitativa por lo último acontecido con la emergencia sanitaria, por ende, los resultados sufrieron varios cambios en el transcurso del desarrollo de la presente investigación, en primera instancia, según se fue recolectando la información, se utilizó el método documental con alcance descriptivo, a esto Hernández Sampieri \& Mendoza, (2018) sustenta que "... tienen como finalidad especificar propiedades y características de conceptos, fenómenos, variables o hechos en un contexto determinado".

Se partió de la recolección y análisis de información documentada; especialmente se utilizó legislación, normativas, decretos, estatutos que rigen al sistema educativo superior, como es el caso de las universidades y escuelas politécnicas públicas con el fin de explicar los dominios científicos, tecnológicos y humanísticos (...) con la colectividad que se realizó en Ecuador (Bonilla et. al. 2019). Ampliando lo que dice Hernández Sampieri \& Mendoza, (2018), el tipo de investigación documental consiste en identificar, recolectar y analizar bibliografía u otras fuentes escritas que brinden información y/o conocimientos de cualquier realidad que se esté investigando; esta información debe ser recabada de forma selectiva, de modo que, la misma sea de utilidad para el propósito que se persigue en la investigación, con el fin de describir las nuevas normativas transitorias y cómo estas influyen en la adaptación de los dominios científicos, tecnológicos y humanísticos sobre las universidades del Ecuador.

Las instituciones seleccionadas fueron entidades representativas de categoría A y categoría B que tenían un alcance mayoritario de los dominios, a su vez, se consideró el registro digital de información. Aparte de los registros digitales, la colaboración con el personal de planificación y las autoridades que quisieron colaborar con esta investigación, es importante recalcar que en un inicio estuvieron 15 IES correspondientes de la Zona 3,4,5 y 6 de las cuales se presenta los resultados. 
Luego de esto, se explica a través de conversatorios a docentes y alumnos de la Universidad Estatal de Bolívar ¿Qué sienten? ¿Cómo están interactuando? ¿Qué opinan? en este proceso de enseñanza-aprendizaje desde su casa, para ello se escogió un muestreo de casos extremos(Creswell, 2014); (Mills et al., 2009). Este muestreo permitió evaluar las características alejadas a la normalidad o de prototipos del problema en estudio, también nos sirve para analizar el comportamiento complejo tanto de los profesores y estudiantes en la modalidad $100 \%$ online. Bajo la premisa descrita, también se consideró significativo conocer los efectos sobre la adaptación de dominios científicos, tecnológicos y humanísticos en línea, considerando la opinión de docentes y alumnos pertenecientes a la Universidad Estatal de Bolívar, se ejecutó conversatorios, en los cuales se realizaron preguntas abiertas tanto de los efectos positivos, como negativos, de la nueva modalidad de estudio, mediante este proceso se dio libertad a que tanto los estudiantes como los docentes expresen una opinión de los aspectos que consideran importantes sobre el nuevo modo de estudio.

\section{Resultados}

En función a la Planificación Nacional en la educación superior se establece direccionar las acciones hacia tres macro procesos realmente importantes como es la Institucionalidad Democrática, Economía Social y Solidaria y Hábitat Sustentable (Figura 4), aspectos que hasta la actualidad son considerados los núcleos potenciadores del buen vivir, surgiendo los principales temas estratégicos direccionados a los problemas sociales que aparecen a nivel nacional, atribuyendo la gestión a las instituciones de educación superior tanto públicas y privadas (Guillen et al., 2015).
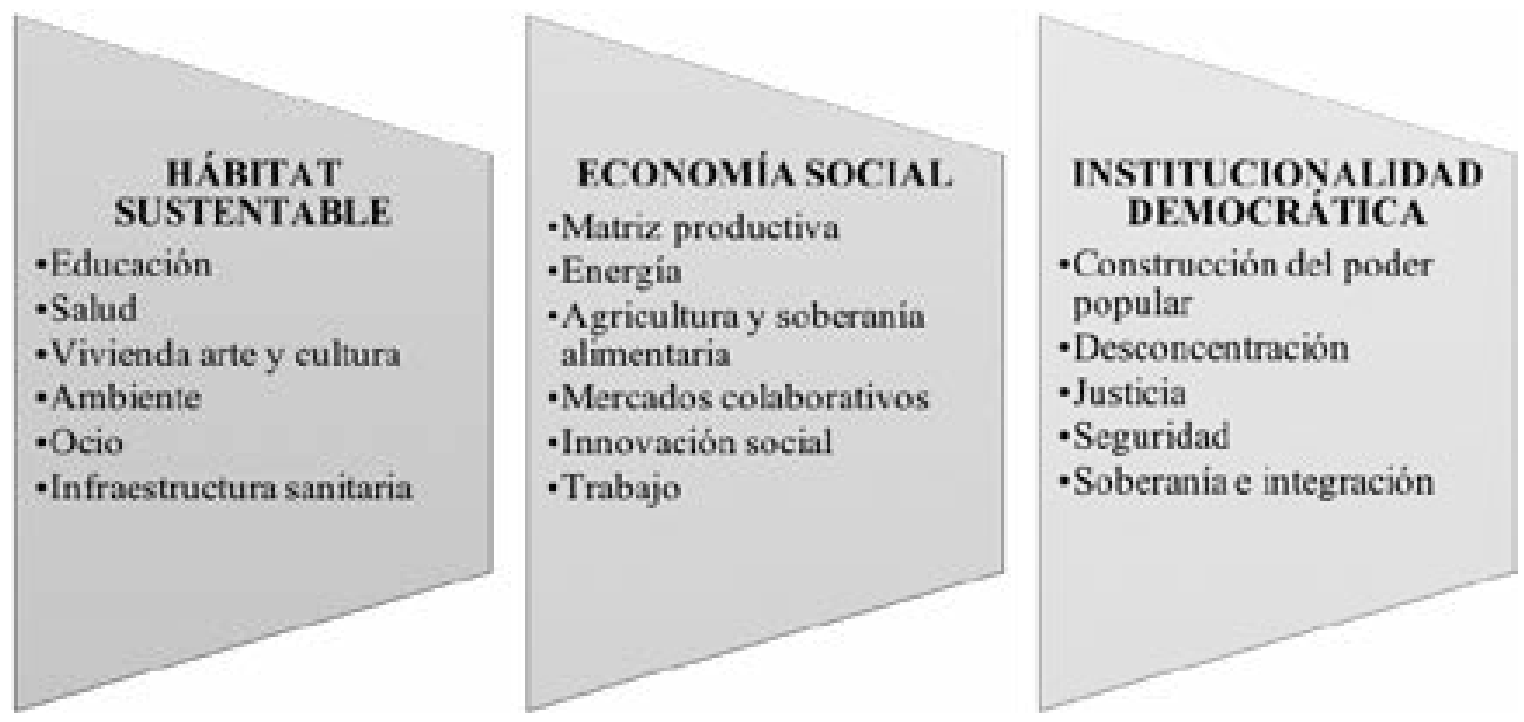

Figura 4. Núcleos potenciadores del Buen Vivir

Fuente: Información obtenida del "Plan estratégico institucional 2015-2019" Universidad San Gregorio de Portoviejo

En la Tabla 1 que se verá a continuación se presenta el listado de universidades con las que se realizó la investigación y los dominios manejados. 
Tabla 1.

Dominios técnicos, científicos, humanísticos de las universidades ecuatorianas

\begin{tabular}{|c|c|}
\hline Universidad & Dominios técnicos, científicos, humanísticos \\
\hline Universidad Técnica de Machala & $\begin{array}{ll}\text { - } & \text { Producción de alimentos } \\
\text { - } & \text { Protección del medio ambiente } \\
\text { - } & \text { Perfeccionamiento de educación superior } \\
\text { - } & \text { Ordenamiento territorial } \\
\text { - } & \text { Ecosistema de salud humana } \\
\text { - } & \text { Ciudadanía local } \\
\text { - } & \text { Desarrollo económico } \\
\text { - } & \text { Nuevas tecnologías }\end{array}$ \\
\hline Universidad Técnica Estatal de Quevedo & 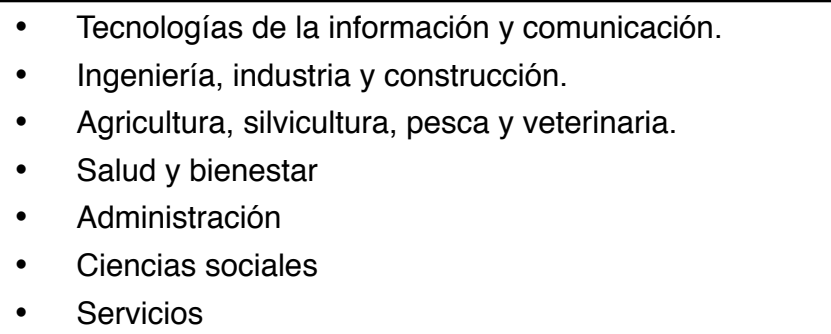 \\
\hline Universidad Técnica de Ambato & $\begin{array}{l}\text { - } \quad \text { Fortalecimiento Social Democrático y Educativo } \\
\text { - Optimización de los sistemas productivos y desarrollo } \\
\text { urbanístico } \\
\text { - Desarrollo económico, productivo y sostenible en } \\
\text { PYMES y MICROPYMES } \\
\text { - Sistemas Alimentarios, Nutrición y Salud Pública }\end{array}$ \\
\hline Universidad de Cuenca & $\begin{array}{l}\text { - } \quad \text { Salud y bienestar humano } \\
\text { - } \quad \text { Ciudad, territorio, infraestructura y patrimonio } \\
\text { - } \quad \text { Tecnologías, industrial y producción } \\
\text { - } \quad \text { Energía, medio ambiente, recursos naturales y biotec- } \\
\text { nología } \\
\text { - Economía, administración y servicios } \\
\text { - Educación, cultura y sociedad }\end{array}$ \\
\hline Universidad Nacional de Chimborazo & $\begin{array}{l}\text { - } \quad \text { Salud como producto social orientado al buen vivir } \\
\text { - } \quad \text { Innovación socio-educativa } \\
\text { - Hábitat sustentable seguro para el desarrollo territorial } \\
\text { - Ingenierías para el desarrollo socio tecnológico y pro- } \\
\text { ductivo }\end{array}$ \\
\hline Universidad Estatal de Bolívar & 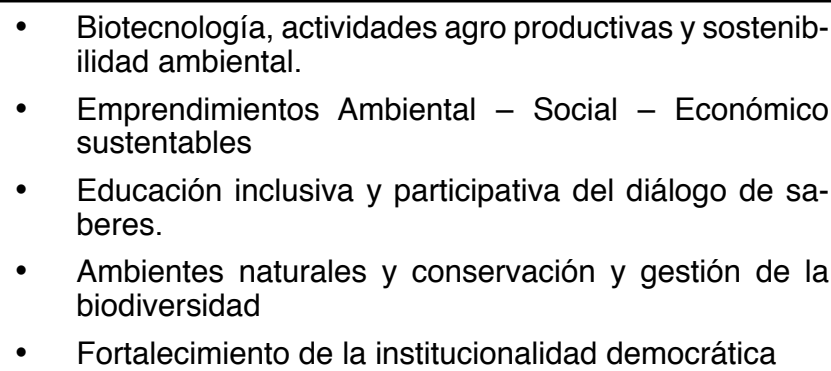 \\
\hline
\end{tabular}

Fuente: elaboración propia 
Mientras que en la Tabla 2 se evidencia una lista enlazada que se utilizó para mostrar los efectos que está generando la adaptación de estos nuevos dominios científicos, tecnológicos y humanísticos en línea, dividido en categorías y subcategorías.

Tabla 2.

Efectos sobre la adaptación de dominios científicos, tecnológicos y humanísticos en línea

\begin{tabular}{|c|c|}
\hline \multirow[t]{5}{*}{ Efectos positivos } & $\begin{array}{l}\text { - Optimización potencial del docente en la conducción de los cursos virtuales, brindados } \\
\text { por la universidad, con la auto preparación previa del maestro. }\end{array}$ \\
\hline & Los estudiantes no se contagian, permanecen en casa. \\
\hline & $\begin{array}{l}\text { Los estudiantes, aprovechan de mejor manera el tiempo programado para activi- } \\
\text { dades, ya que no pierden clases, evitan accidentes, ahorran recursos económicos de } \\
\text { su transporte. }\end{array}$ \\
\hline & $\begin{array}{l}\text { - Los estudiantes se ven obligados a fortalecer su capacidad lectora porque la modali- } \\
\text { dad virtual propende más al trabajo autónomo. }\end{array}$ \\
\hline & Los estudiantes fortalecen su innata capacidad de abstracción, análisis y síntesis. \\
\hline \multirow[t]{4}{*}{$\begin{array}{l}\text { Efectos } \\
\text { negativos }\end{array}$} & $\begin{array}{l}\text { - Estrés en docentes y estudiantes por trabajo virtual, sumado al estrés de la crisis san- } \\
\text { itaria. }\end{array}$ \\
\hline & $\begin{array}{l}\text { - En docentes, problemas de salud, columna, espalda, visión, a ello se suman las difi- } \\
\text { cultades existentes }\end{array}$ \\
\hline & El trabajo virtual se duplicó para el maestro y estudiante. \\
\hline & $\begin{array}{l}\text { - Los estudiantes se quejan de poca explicación en los temas por parte de sus do- } \\
\text { centes y el envío excesivo de trabajos en casa. }\end{array}$ \\
\hline
\end{tabular}

Fuente: elaboración propia

\section{Conclusiones}

Con base en los resultados se destacan los dominios cultura, educación y economía, los mismos que aportan al cumplimiento de lo establecido en el Plan de Desarrollo Toda una Vida, comprendiendo así que, al darse este cumplimiento, se genera un eje relevante en la planificación estratégica de las instituciones.

En la línea de tiempo se pueden analizar dos momentos, el primero es el de la pre-pandemia, donde las IES mostraban ya sustento en el cumplimiento de lo planteado en el Plan Nacional de Desarrollo -Toda una Vida, fortaleciendo los núcleos principales del Buen Vivir que son: institucionalidad democrática, economía social y solidaria y hábitat sustentable; en función a estos núcleos se conoce que, de las 6 universidades evaluadas, todas concuerdan con que deben aún potenciar los aspectos tecnológicos.

Del mismo modo, otro aspecto a destacar entre la concordancia de las instituciones es el de la innovación social, esto sin olvidar la calidad en la planificación y dirección para que se cumplan metas y objetivos que es a lo que obedece todo el sistema de operación. Y el segundo momento que es el de la post pandemia, compromete a las IES a intensificar sus actividades formativas a través de la virtualidad. Esto ha desencadenado una serie de ventajas y desventajas en el proceso enseñanza - aprendizaje, pero también, impulsa a una transformación en los dominios científicos, tecnológicos y humanísticos que fungen de ayuda a los procesos sustantivos de docencia, 
investigación y vinculación de las instituciones para la solución de los diversos problemas que están presentándose con esta crisis.

Sin duda, las universidades tienen que fortalecer estos dominios, identificando con claridad aquellos recursos humanos idóneos, para que, con la mayor precisión y agilidad posible, trasmitan al resto de instancias académicas sus conocimientos y estos coadyuven al definitivo posicionamiento de una nueva cultura en la virtualidad, que refuerce los dominios entre todos los actores de este ecosistema.

Finalmente, el ajuste de los mecanismos de evaluación de los entornos virtuales de aprendizaje y de las clases en línea, deberá trasladarse a la clase invertida, donde el alumno es quien gestiona su aprendizaje y, por ello, el tiempo de clase presencial es usado para debatir, aclarar y trabajar puntos clave, así como cualquier pregunta o dificultad que los estudiantes puedan tener. Potencializar el flipped classroom o clase invertida es muy importante, ya que existen modelos instructivos similares que gozan también de buena reputación. Algunos de ellos pueden ser la instrucción entre pares, o el aprendizaje basado en problemas complementado con el estudio de casos y tecnologías educativas.

Hoy, más que nunca, el principal reto de las IES es acertar en la búsqueda de una alternativa que consolide la práctica docente en línea e integre en las Tecnologías de la Información y la Comunicación (TIC) un recurso de soporte centrado en la presencialidad. Sin embargo, cuando los estudiantes tienen que interactuar en los espacios de formación en línea de forma constante, es imperativo un alto nivel de desarrollo de las competencias digitales en los profesores. Entonces, es aquí, cuando los docentes debemos intensificar nuestra autoformación, optimizar el tiempo y adaptar la práctica pedagógica a la nueva aula virtual. La consideración de cómo se enseña y aprende en las universidades está directamente relacionada con el nivel de formación profesional de los facilitadores. 


\section{Referencias}

Asamblea Nacional del Ecuador. Ley Orgánica de Educación Superior (LOES). Registro Oficial Suplemento No 298, (2010). https://www.siteal.iiep.unesco.org/sites/default/files/sit accion files/ec 6011.pdf

Asamblea Nacional del Ecuador. Ley Orgánica Reformatoria de la Ley Orgánica de Educación Superior (LOR LOES). Registro Oficial Primer Suplemento N. 297, (2018). https://www.epn.edu.ec/wp-content/ uploads/2018/08/LOES.pdf

Bonilla, D. M., Ocampo León, C. S., Camacho Escobar, M. A., \& Pinos Morales, G. J. (2019). Estudio legal y estrategias sobre vinculación universitaria con la colectividad, Caso Ecuador. Killkana Social, 3(2), 57-64. https://doi.org/10.26871/killkana social.v3i2.465

CAF Corporación Andina de Fomento. (2020). Las oportunidades de la digitalización en América Latina frente al COVID-19. In Corporación Andina de Fomento. https://repositorio.cepal.org/bitstream/handle/11362/45360/4/OportDigitalizaCovid-19 es.pdf

Creswell, J. (2017). Research design: Qualitative, quantitative, and mixed methods approaches. Sage publications. https://search.proquest.com/openview/237a4fa7c4967d1cc1b5e5da8771e0ef/1 ?pq-origsite=gscho$\underline{\text { lar\&cbl}=33100}$

Guillen, X., Farfán, M., Alarcón, L., \& Alarcón, J. (2015). Plan estratégico institucional 2015-2019. http://planificacioninstitucional.sangregorio.edu.ec/plan-estrategico/

Hernández, R., \& Mendoza, C. (2018). Metodología de la investigación: las rutas cuantitativa, cualitativa y mixta (1st ed.). McGraw Hill. http://virtual.cuautitlan.unam.mx/rudics/wp-content/uploads/2019/02/RUDICSv9n18p92 95.pdf

Larrea, E., \& Granados, V. (2013). El sistema de educación superior para la sociedad del buen vivir basada en el conocimiento: El caso ecuatoriano [Universidad Católica Santiago de Guayaquil]. http://www.uti.edu. ec/ utiweb/wp-content/uploads/2019/05/Dominios de Investigación-compressed.pdf

Maldonado, B. (2017). Gestión Universitaria y formación por competencias. In D. Andrade (Ed.), Aprendizaje y formación valorica en la enseñanza mediante tutoriads entre pares (Vol. 16, Issue 3). https://repositorio. espe.edu.ec/bitstream/21000/11681/1/Gestion Universitaria.pdf

Mills, A., Durepos, G., \& Wiebe, E. (2009). Enciclopedia of case study research. Sage Publications, Inc. https:// archive.org/details/2.encyclopediaOfCaseStudyResearch/page/n3/mode/2up

Mora, E., Bonilla, D., Núñez, L., \& Sarmiento, J. (2018). Inadaptabilidad de los docentes al manejo de plataformas virtuales: caso educarecuador. Revista Pedagógica de La Universidad de Cienfuegos, 14(62), 1-5. http://scielo.sld.cu/pdf/rc/v14n62/rc066218.pdf 
Morales, M. (2012). Prácticas de resistencia docente en la universidad orientada al mercado: Capturas y fugas académicas en el contexto colombiano [Universidad Autónoma de Barcelona]. https://www.tdx.cat/bitstream/handle/10803/107904/mdmh1de1.pdf?sequence=1\&isAllowed=y

Normativa transitoria para el desarrollo de actividades académicas en las Instituciones de Educación Superior. RPC-SE-03-No 046-2020, (2015). https://www.epn.edu.ec/wp-content/uploads/2020/04/normativa transitoria rpc-se-03-no.046-2020.pdf

Pineda, L. (2013). Prospectiva estratégica en la gestión del conocimiento: una propuesta para los grupos de investigación colombianos. Investigación \& Desarrollo, 21(1), 289-311. http://www.scielo.org.co/scielo. php?pid=S0121-32612013000100010\&script=sci abstract\&tlng=pt

Ramírez, R. (2016). Universidad urgente para una sociedad emancipada (Primera ed). Senescyt \& lesalc, Unesco. https://revistachasqui.org/index.php/chasqui/article/view/2897/2760

Rivadeneira, E. (2016). El I Congreso de Ciencia y Tecnología como estrategia para fortalecer la publicación científica en la Universidad Técnica de Machala. Yachana Revista Cientifica, 5(1), 121-129. http://repositorio.ulvr.edu.ec/bitstream/44000/3706/1/EI I Congreso de Ciencia y Tecnología como estrategia para fortalecer la publicación científica en la Universidad Técnica de Machala.pdf

Rojo, M. A., \& Bonilla, D. M. (2020). COVID-19: La necesidad de un cambio de paradigma económico y social. CienciAmérica, 9(2), 77. https://doi.org/10.33210/ca.v9i2.288

Ruzmar, I., \& Pérez, A. (2017). Diseño de plan estratégico para fortalecer el clima organizacional del personal docente de la facultad de ingeniería en la universidad de Carabobo [Universidad de Carabobo]. http:// www.riuc.bc.uc.edu.ve/bitstream/123456789/4619/1/rperez.pdf

Secretaría Nacional de Planificación y Desarrollo. Plan Nacional de Desarrollo. (2017). Plan Nacional de Desarrollo 2017-2021. Toda una Vida. http://www.planificacion.gob.ec/wp-content/uploads/downloads/2017/10/ PNBV-26-OCT-FINAL OK.compressed1.pdf 
Copyright (c) 2021 Florcita Janeth Arellano Espinoza, María de los Ángeles Bonilla, Jonathan Patricio Cárdenas Benavides y Danny Fernando Pérez Castillo

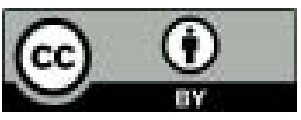

Este texto está protegido bajo una licencia internacional creative Commons 4.0.

Usted es libre para Compartir-copiar y redistribuir el material en cualquier medio o formato

- y Adaptar el documento - remezclar, transformar y crear a partir del material-para cualquier propósito, incluso para fines comerciales, siempre que cumpla las condiciones de Atribución. Usted debe dar crédito a la obra original de manera adecuada, proporcionar un enlace a la licencia, e indicar si se han realizado cambios. Puede hacerlo en cualquier forma razonable, pero no de forma tal que sugiera que tiene el apoyo del licenciante o lo recibe por el uso que hace de la obra.

\section{$\underline{\text { Resumen de licencia - Texto completo de la licencia }}$}

